\title{
Archéopages
}

Archéopages

Archéologie et société

Hors-série 2 | 2010

Archéologie sans frontières

\section{Les fouilles de l'aéroport de Siem Reap à Angkor}

\section{Pierre Bâty}

\section{OpenEdition}

\section{Journals}

Édition électronique

URL : https://journals.openedition.org/archeopages/791

DOI : 10.4000/archeopages.791

ISSN : 2269-9872

\section{Éditeur}

INRAP - Institut national de recherches archéologiques préventives

\section{Édition imprimée}

Date de publication : 1 octobre 2010

Pagination : 86-93

ISSN : 1622-8545

\section{Référence électronique}

Pierre Bâty, «Les fouilles de l'aéroport de Siem Reap à Angkor », Archéopages [En ligne], Hors-série 2 |

2010, mis en ligne le 01 octobre 2010, consulté le 23 février 2023. URL : http://

journals.openedition.org/archeopages/791 ; DOI : https://doi.org/10.4000/archeopages.791 

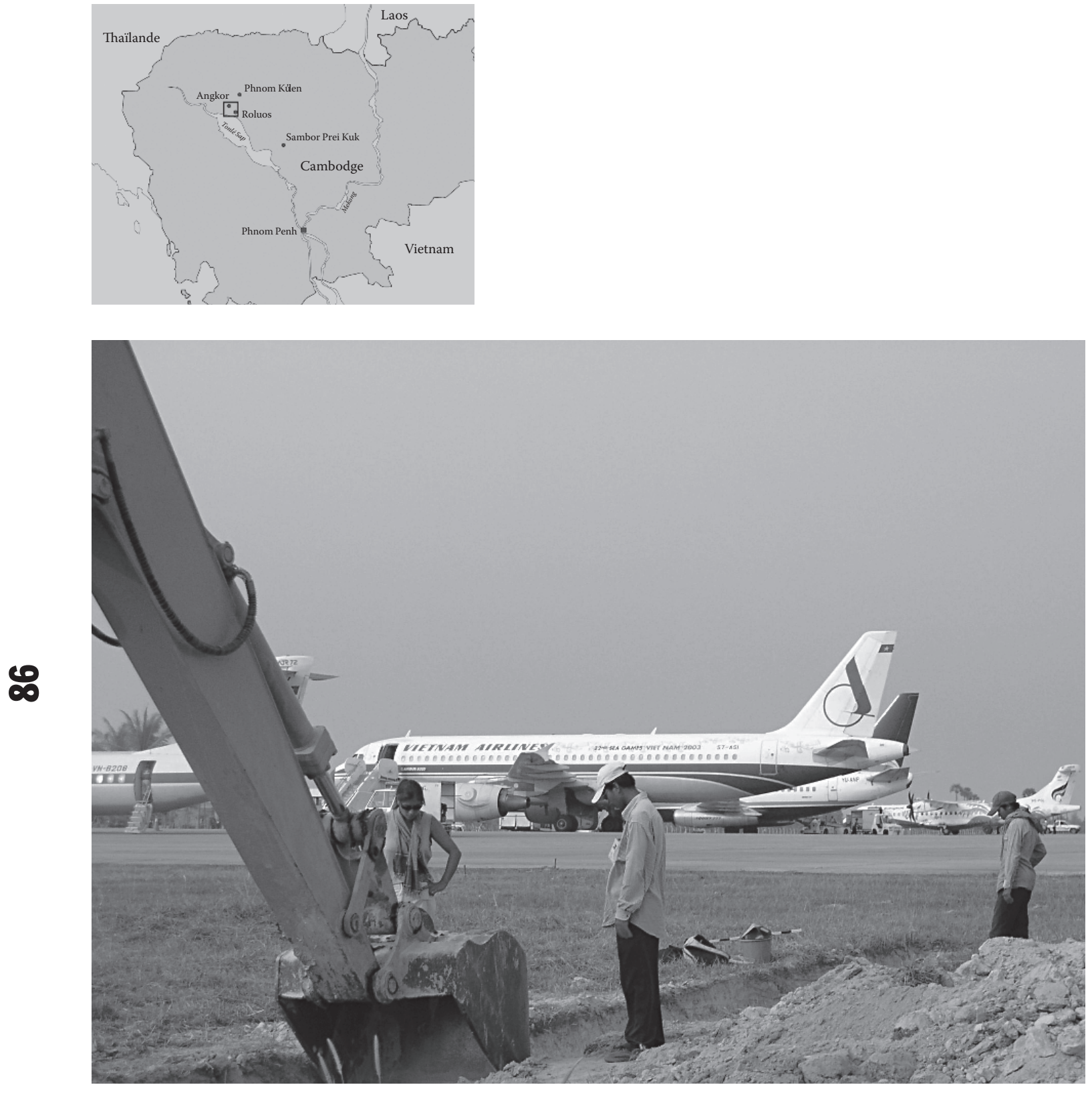

[Fig. 1] Vignette de localisation.

[Fig.2] Diagnostic en cours à l'aéroport. 


\section{Les fouilles de l'aéroport de Siem Reapà Angkor}

Pierre Bâty

Inrap

A ngkor ne se résume pas à Angkor Vat, à l'inverse de ce que l'imaginaire occidental retient trop souvent. C'est un site archéologique immense (près de 40 ooo ha) qui n'est pas un simple site-musée [Fig.1]. Angkor est aussi un lieu vivant, toute une population y habite, y travaille, y consomme, s'y déplace. Le tourisme, en pleine expansion, entraîne un aménagement du territoire et une pression foncière importante. Ces flux, comme cette réalité démographique, ont un impact certain sur la conservation de sites fragiles, qu'ils soient monumentaux ou pas.

Le site de l'aéroport de Siem Reap à Angkor est localisé dans le périmètre de la zone 1 , inscrite au Patrimoine mondial de l'Unesco et faisant l'objet d'une protection maximale. Il est géré par une filiale du Groupe Vinci, la SCA $\mathbf{1}$, qui administre également les aéroports de Phnom Penh et de Sihanoukville. C'est à l'occasion de la construction du nouveau terminal international que des fouilles préventives ont été réalisées en 2004, financées intégralement par la SCA, en partenariat avec l'APSARA $^{3}$ et l'Inrap.

L'étude des photographies aériennes de la zone d'implantation de l'aéroport montre une quantité de tertres d'habitat, de sanctuaires, de structures linéaires et d'indices parcellaires, appartenant à la période angkorienne et pré-angkorienne. L'installation du champ d'aviation en 1931 avait déjà motivé une première opération archéologique sur le site de Prasat Trapeang Ropou [Fig.3 à 5], par l'EFEO ${ }^{4}$ sous la direction d'Henri Marchal (Marchal, 1931). Des travaux d'agrandissement furent ensuite menés à partir de 1939, jusqu'en 1946. Ils concernent la construction d'une première piste en béton. En 1966, l'extension de la piste est entreprise. Ces travaux, ainsi que les combats livrés autour de l'aéroport dans les années 1980 et 1990, ont gravement nuit aux sites archéologiques de cette zone. Il faut attendre 1998 pour que l'APSARA et la direction de l'Aviation civile demandent une évaluation du potentiel archéologique de l'aéroport (Gaucher, Franiatte, 1999). Cette démarche novatrice n'a pas empêché de nouveaux terrassements destructeurs d'avoir lieu en 1999 et en 2000. C'est donc dans un environnement très dégradé que nous avons dû intervenir en 2004.

Le diagnostic archéologique de l'aéroport international de Siem Reap [Fig.2], sur une superficie de 26 hectares, a permis d'aborder deux sites angkoriens datés des $\mathrm{X}^{\mathrm{e}}$ et $\mathrm{XI}^{\mathrm{e}}$ siècles : Prasat Trapeang Ropou et Trapeang Thlok (cf. encadrés p. 88 et 89 ). La fouille exhaustive, sur plus de 2 ha du site de Trapeang Thlok est une première qui permet d'observer et d'analyser l'organisation d'un site d'habitat et d'un sanctuaire associés. Une première étude typo-chronologique de la céramique a été réalisée en tenant compte de l'ensemble du vaisselier (Desbat, dans Bâty, 2005).

Ce travail fait partie des rares fouilles exhaustives menées au Cambodge à ce jour. La réussite de cette opération archéologique et les questionnements qu'elle a suscités ont conduit à une réflexion sur la poursuite des recherches dans le secteur de l'aéroport. Ce nouveau projet scientifique, programmé sur cinq ans, aborde en priorité la question de l'habitat et de son insertion dans les trames foncières et territoriales, puisqu'il est connecté à un groupe de temples mitoyens, remontant aux $\mathrm{X}^{\mathrm{e}}-\mathrm{XII}^{\mathrm{e}}$ siècles. La chronologie de ces occupations reste à préciser.

Des états de parcellaires angkoriens. Les contraintes morphologiques majeures qui caractérisent ce secteur d'Angkor appartiennent à des époques variées et traduisent une longue occupation de la zone. Les principales contraintes correspondent à des digues et des canaux : le Baray occidental, gigantesque réservoir daté $\mathrm{du} \mathrm{XI}^{\mathrm{e}}$ siècle, la double digue dite de «Yaçodharapura», reliée au Baray et à l'ensemble des structures pré-angkoriennes qui constituent le site de «Banteay Choeu» (Groslier, 1974). Dans ce maillage structurant se développent différents états de parcellaires asynchrones. Ce système de rizières délimitées par des digues est irrigué par gravité depuis le Baray occidental. Il est admis qu'une partie au moins de ce système soit contemporaine du Baray et donc datée du XI ${ }^{\mathrm{e}}$ siècle (Pottier, 1999).

Juste au sud du temple de Trapeang Thlok nous avons coupé une trentaine de digues de rizières. Elles sont organisées selon un axe approximativement nord-sud et est-ouest, mais leur orientation varie de quelques degrés. Cet écart se manifeste par une disposition « en éventail » de certains groupes de parcelles, qui tranche par rapport à l'orthogonalité de l'organisation générale et suggère que la zone étudiée est située aux confins de plusieurs espaces fonciers. La disposition des sanctuaires voisins accrédite cette hypothèse.

Ces digues se superposent parfois à de petits fossés plus anciens dont elles reprennent le tracé. Des sols culturaux recouvrent les digues des parcellaires dont ils atténuent le relief. Ils contiennent très peu de mobilier archéologique : quelques tessons de céramique commune très érodés et des grès angkoriens. Un élément chronologique nous est toutefois fourni par la découverte d'une occupation $\mathrm{du} \mathrm{XI}^{\mathrm{e}}$ siècle superposée à une digue de parcellaire.

Grâce aux photo-interprétations et aux observations au sol, les limites entre les groupes parcellaires afférents à Prasat Trapeang Ropou et Trapeang Thlok ont pu être déterminées. Ces interactions mettent en évidence des agrégats de parcelles, accolées aux limites préexistantes de groupes fonciers plus anciens (Bâty, 2005).

Un nouveau projet, des thématiques cohérentes. L'existence d'un patrimoine monumental sur le foncier même de l'aéroport et à ses abords immédiats motive aujourd'hui une programmation archéologique pluriannuelle, de l'aéroport.

Autorité pour

de la région d'Angko

4 École française

d'Extrême-Orient. 
établie sur cinq ans, consacrée à la fouille des sites localisés dans le domaine sécurisé de l'aéroport et en périphérie. Ces fouilles s'inscrivent dans une problématique scientifique centrale : leur étude exhaustive permettra, dans la suite des travaux de 2004, d'aborder pleinement la question de l'habitat angkorien, sa forme, son évolution, et les interactions entre plusieurs groupes d'habitats dans une période comprise entre le $\mathrm{X}^{\mathrm{e}}$ et le $\mathrm{XII}^{\mathrm{e}}$ siècle. Ces fouilles serviront de chantiers d'application pour la formation de jeunes étudiants cambodgiens en archéologie. Elles serviront à la mise en place de « classes culturelles » en direction des écoles de la zone. Afin de permettre à l'APSARA de disposer des nouveaux cadres nécessaires au développement de son service d'archéologie préventive, un cycle de formation aux techniques de l'archéologie préventive sera développé dans le cadre de ces fouilles. Cette démarche est aujourd'hui ressentie comme une nécessité, tant par les futurs ingénieurs/archéologues cambodgiens que par les institutions internationales qui font appel à eux. Cette formation doit être proposée aux jeunes étudiants en archéologie comme un complément à leur formation universitaire.

Une telle démarche a déjà été initiée, puisque des agents de l'Apsara ont pu bénéficier d'une formation de trois mois au Centre archéologique de l'Inrap à Poitiers. Ils ont été intégrés à plusieurs opérations d'archéologie préventive, et ont suivi une formation de la part de spécialistes de l'établissement et de l'Université de Poitiers.5 Ce chantier-école pourra devenir le laboratoire où seront adaptées, aux spécificités de l'archéologie angkorienne, les méthodes de terrain et d'enregistrement. Il est important, dans le contexte du patrimoine mondial, que l'APSARA puisse bénéficier d'un tel outil. La formation se fera sur le terrain, dans le cadre du programme de fouille pluriannuel.

Le nouveau programme de fouille. Huit sanctuaires associés à de l'habitat et à des bassins sont concernés par le projet, ils présentent des cohérences géographiques et foncières (sites mitoyens dont les espaces fonciers interagissent), thématiques (présence de tertres, de terre-pleins, de trapeang, de bassins inscrits dans des espaces afférents) et chronologiques (fondations supposées $\mathrm{au} \mathrm{X}^{\mathrm{e}}$ siècle ou au début du $\mathrm{XI}^{\mathrm{e}}$ siècle, pérennité de l'occupation durant les $\mathrm{XI}^{\mathrm{e}}$-XII ${ }^{\mathrm{e}}$ siècles au moins). De ces huit sites, seuls trois ont fait l'objet de recherches archéologiques par le passé : le grand ensemble Prasat Preï (Trouvé, 1933), Prasat Trapeang Ropou (Marchal, 1931) et récemment Trapeang Thlok. Les autres sites ont été prospectés par Ch. Pottier (EfEO) (Pottier, 1999) et par Pierre Bâty de 2004 à 2010.

C'est en 1933 que Georges Trouvé (EFEO) entreprend le dégagement de la tour centrale de Prasat Preï. Plusieurs éléments statuaires sont découverts, dont une représentation de Brahma en grès attribuable au premier quart $\mathrm{du} \mathrm{XI} \mathrm{I}^{\mathrm{e}}$ siècle, aujourd'hui exposée au Metropolitan Museum of Art de New York ${ }^{\mathbf{6}}$. Ce temple en brique est installé sur une plate-forme de 100 m de côté, entourée de douves et associé à un grand trapeang [Fig.9]. Le site se développe sur 700 m environ selon un axe est-ouest rigoureux et s'insère dans un parcellaire isocline en contact avec l'espace foncier attribué au Prasat Trapeang Thlok, fouillé en 2004.

Le terre-plein du sanctuaire présente une dépression carrée dans son angle nord-est correspondant à un petit bassin. Plusieurs microreliefs, dont il reste à définir l'origine, marquent la plate-forme. En 2009, nous avons pu faire réaliser le débroussaillage du sanctuaire. Après nettoyage, la tour centrale ressort nettement. En briques, elle ne présente pas d'éléments de datation évidents, notamment aucun caractère typiquement pré-angkorien. La décoration est inachevée. Une seconde tour inédite, très arasée, apparaît au nord de la première. L'ensemble pourrait se situer au début $\mathrm{du} \mathrm{x}^{\mathrm{e}}$ siècle. Nous ne possédons aucun élément de datation pour les nombreux tertres associés à cet ensemble au sud et à l'ouest, ni sur les occupations probables des remblais entourant le bassin, propices à l'installation de villages. Il est vraisemblable que l'occupation se soit poursuivie sur une assez longue période.

Plusieurs sites apparaissent très dégradés ou menacés par les terrassements liés aux aménagements de l'aéroport. C'est notamment le cas de Tuol Ta Lo, coupé en deux par la piste de secours. Ce sanctuaire se compose d'un terre-plein, entouré de douves et ouvert à l'est par une chaussée, de deux petits bassins et d'un tertre au nord. L'ensemble se développe sur plus de $200 \mathrm{~m}$ selon un axe est-ouest. En 2004, lors de notre intervention, un beau piédestal en grès y a été découvert, il gisait en bordure de la piste de secours. Il est aujourd'hui déposé au musée Norodom Sihanouk à Siem Reap.

L'extension de la piste a détruit un sanctuaire connu grâce aux photographies aériennes de W. Hunt en 1945 (Anonyme, CP 707 : cf. Pottier, 1999). L'ensemble se développe sur $360 \mathrm{~m}$ selon un axe est-ouest et comprend un trapeang, un terre-plein et un tertre (habitat?). En mai 1966, les terrassiers y ont découvert une cache d'objets en bronze comprenant de nombreuses divinités, attribuées au XIII ${ }^{\mathrm{e}}$ siècle. Les 44 objets recensés dans ce lot font aujourd'hui partie des plus belles pièces en bronze du Musée national à Phnom Penh.

Localisé à l'extrémité sud-ouest de la piste, le site de Kôk Neak Ta Kruong est coupé en deux par la clôture de l'aéroport. Il possède un trapeang (bassin sacré), prolongé à l'ouest par un terre-plein entouré de douves, puis par un très grand tertre de $100 \mathrm{~m}$ de long. Des tertres plus petits, localisés au sud du sanctuaire, peuvent être rattachés à cet ensemble. Quelques éléments lapidaires (linteau, piédroits) attestent d'une architecture à la décoration soignée.

Le site de Kôk Russeï correspond à un trapeang d'axe est-ouest, aujourd'hui très dégradé. Un tertre au nord possède un beau piédestal en grès décoré sur chaque face d'un petit personnage dans une arcature et des éléments d'architecture [Fig.10]. de Poitiers.

Southeast Asia», The

Metropolitan Museum of Art Bulletin, vol. 51, $\mathrm{n}^{\circ} 4$. 

Il a été vandalisé dernièrement, nous avons demandé à l'APsARA sa mise à l'abri. Une zone surélevée, à l'ouest du trapeang, particulièrement dévastée par les prises de terre, pourrait correspondre à un tertre de grande dimension, ou aux vestiges du terre-plein d'un temple. L'ensemble se développe sur plus de $270 \mathrm{~m}$ selon un axe est-ouest. Lors de nos prospections, de la céramique post-angkorienne a également été observée à l'ouest du site, suggérant une occupation assez longue.

Le site de Trapeang Samrong est composé d'un petit terre-plein entouré de douves, d'un gros tertre quadrangulaire, au sud, accompagné d'un tertre secondaire, d'une chaussée axiale et de quatre petits bassins. L'ensemble est complété à l'est par un très beau trapeang. Certaines anomalies topographiques évoquent l'existence d'un grand enclos fossoyé, mais ce point reste très hypothétique. Des prises de terre en 2005 ont provoqué la destruction du gros tertre au sud du terre-plein. Les prospections sur p lace ont permis de récolter un peu de céramique angkorienne. La présence de grès à glaçure brune associée à d'autres fragments de type Kulen suggère une occupation s'étendant au moins jusqu'au $\mathrm{XII}^{\mathrm{e}}$ siècle. Les travaux de construction d'une route effectués en 2005 ont gravement altéré le site, qui est aujourd'hui menacé de disparition.

Problématique et objectifs scientifiques. L'état de la connaissance des sites intégrés dans ce projet est, comme nous l'avons vu, très inégal : Prasat Trapeang Ropou est assez bien documenté. Les analyses radiométriques, l'étude de la céramique comme l'approche stylistique et la traduction des inscriptions confirment une installation probable sous le règne du souverain Rajendravarman au milieu du $\mathrm{X}^{\mathrm{e}}$ siècle, l'occupation se poursuivant jusqu'au milieu du XII ${ }^{\mathrm{e}}$ siècle au moins. Pour les autres sites, nous n'avons que des connaissances sporadiques et lacunaires. Leur datation est souvent indirecte, jamais précise, sauf à Trapeang Thlok grâce aux analyses radiométriques et à l'étude de la céramique.

Deux périodes plus ou moins distinctes semblent se dessiner : des fondations au $\mathrm{x}^{\mathrm{e}}$ siècle, peut-être précoces, comme Trapeang Ropou. Ces ensembles s'inscrivent dans des espaces fonciers homogènes et isoclines. Des fondations plus modestes ne présentant pas de vestiges monumentaux et dont les espaces fonciers, moins homogènes, semblent accolés aux limites préexistantes des gros ensembles du $\mathrm{x}^{\mathrm{e}}$ siècle. En l'état actuel des connaissances, ces groupes ne remonteraient pas au-delà de la fin du $\mathrm{x}^{\mathrm{e}}$ siècle. Ces décalages chronologiques et morphologiques sont à l'origine du questionnement motivant ce programme de recherches. Ils posent la question du statut de ces différents sites, de leurs habitants et de leurs interactions.

Parcellaire et espace foncier. Cette approche systématique sur un groupe de temples et d'habitats limitrophes nous apportera les éléments de datation essentiels pour comprendre l'évolution de la zone et la construction du paysage. Les résultats de ces opérations archéologiques permettront d'étudier les interactions de plusieurs sanctuaires de dimensions et de statuts différents (simples temples en bois ou édifices en briques), associant de l'habitat (village?) à des « territoires immédiats $»$.

Le terme d' « espace foncier » reste à pondérer : il renvoie à des notions fiscales et/ou juridiques avérées dans le cas de Prasat Trapeang Ropou (querelle de bornage entre Ropou et Vnam Kantal : inscription K 690), mais purement spéculatives en l'état des connaissances pour les autres sites. Ces territoires correspondent à un ancien état d'appropriation de l'environnement, centré sur des temples, dont la mesure pourrait être considérée comme la distance de la maison aux champs. À l'intérieur de ces espaces, la dispersion de l'habitat ainsi que la forme et la contenance des parcelles paraissent induites par des contraintes techniques liées aux pratiques agricoles et au caractère diachronique de ces ensembles parcellaires entre eux. À ce stade du raisonnement, l'apport de la géo-archéologie, combinant une analyse géomorphologique et pédologique, est important. L'étude géomorphologique nous renseignera sur les choix d'implantation des habitations au regard des ressources naturelles, hydriques et agronomiques, du milieu.

Parallèlement, l'étude pédologique permettra de faire la part des caractéristiques naturelles des sols et celles héritées de son anthropisation. Il est donc crucial, afin de déterminer quelques-unes de ces interactions, de pouvoir sonder mécaniquement des intersections de digues et de diguettes offrant le plus de chance de livrer des informations sur ce phasage et ces contraintes. L'étendue des investigations correspond à une zone de 10,55 km² intégrant l'aéroport et les terrains afférents. En complément de ces sondages ciblés, des relevés topographiques au sol, une couverture photographique aérienne et l'utilisation du LIDAR (Light Detection and Ranging), permettront la réalisation d'un modèle numérique de terrain précis, illustrant cette organisation du territoire.

Sanctuaires et habitats. À l'échelle des sites étudiés, les résultats des fouilles des tertres permettront d'aborder les questions liées à la constitution et à l'organisation de l'habitat d'un point de vue technique, spatial et social, à l'intérieur de plusieurs phases chronologiques. L'un des points éminents de ce programme est l'étude du lien structurel entre le temple et l'habitat, tant du point de vue de la culture matérielle que de la structuration géographique et physique de l'ensemble. Il est nécessaire de rappeler qu'en un siècle d'archéologie à Angkor, les questions liées à l'habitat n'ont que très rarement été abordées.

La fouille des sanctuaires et des habitats permet d'espérer apporter des connaissances nouvelles sur l'architecture en matériaux périssables, à l'image du temple en bois de Trapeang Thlok. La fouille exhaustive de plusieurs tertres d'habitat et 

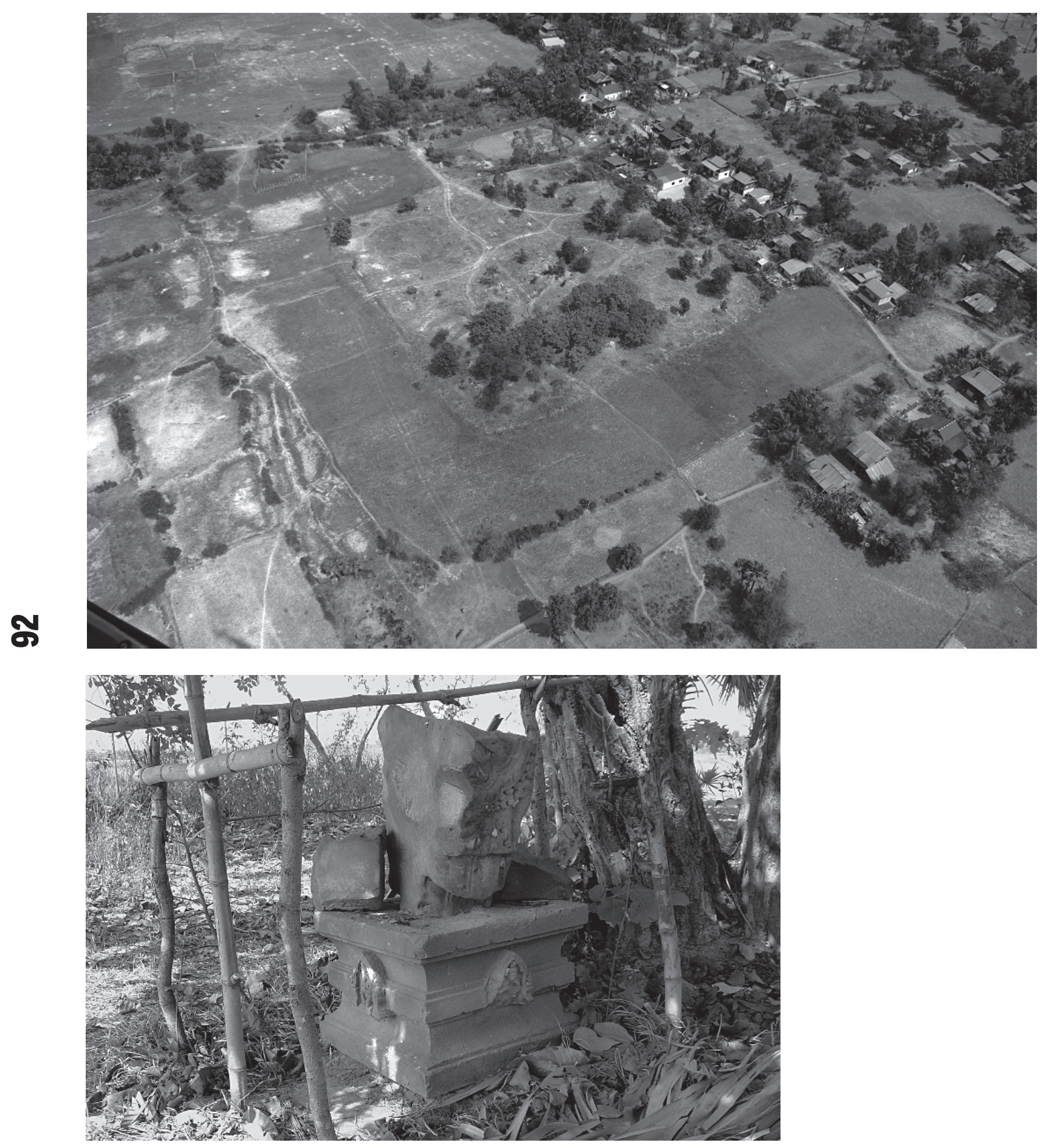

[Fig.9] Vue aérienne du temple et des douves de Prasat Preï.

[Fig.10] Piédestal de Kôk Russeï. 
les sondages sur les berges des grands trapeang permettront d'aborder la question de l'habitat de manière globale, en menant une réflexion sur l'organisation, le déplacement et l'extension de celui-ci autour des différents sanctuaires. Le projet vise une approche globale, considérant le temple et le village à l'intérieur d'un territoire, lui-même influencé par des limites préexistantes et contraignantes.

BÂTy P., Bolle A., novembre 2005, "Sanctuaires et habitats sous l'aéroport de Siem Reap, Cambodge », Archéologia, n² 427, p. 18-23.

BÂTy P. et al, 2005, «Extension de l'aéroport de Siem Reap, Trapeang Thlok Prasat Trapeang Ropou », Rapport de diagnostic archéologique ; "Trapeang Thlok sanctuaire et habitat », Rapport de fouille, DFS de sauvetage urgent, base Inrap de Poitiers, 1 volume $\mathrm{A}_{3}$ 167 pages, 157 fig., 248 photographies.

BÂTy P., 2007 « Les couteaux angkoriens de Trapeang Thlok et de Prasa Trapeang Ropou », Befeo, 94, p. 95-110.

COEDES G., 1952, Inscriptions du Cambodge, vol. 4, p. 151, Paris, EFEO.

Dagens B., 1994 «Le temple indien en Asie du Sud-Est. Archéologie d'une forme », in BIzOT F. (DIR.), Recherches nouvelles sur le Cambodge, Paris, Efeo, p. 259-272.

GAUCHER J., FrAniatTe M., 1999, Recherches archéologiques à l'aéroport de Siem Reap. Opération Préventive, DFS, Mission archéologique française à Angkor, $157 \mathrm{p}$.

Groslier B. PH., 1979, « La cité hydraulique angkorienne : exploitation ou surexploitation du sol ?», Befeo, 68, p. 161-202.

Groslier B. PH., 1974 « Agriculture et religion dans l'Empire angkorien ", Études Rurales, 53-54-55-56, p. 95-117.

LUNet De LAJONQUiere É. E., 1911, Inventaire descriptif des monuments $d u$ Cambodge, t. III, Efeo, Paris, p. 132

MARCHAL H., 1922 à 1936, Journaux de Fouille - région d'Angkor Archives de l'École française d'extrême orient, Paris.

Marchal H., 1920, Rapport de la conservation d'Angkor, Archives de l'Efeo, Paris.

Pottier Chr., 1999, Carte archéologique de la région d'Angkor zone sud, thèse de doctorat, Université de Paris III, Ufr Orient et Monde Arabe, 3 volumes, 382 p., 33 cartes.

Pottier Chr., 200o, «À la recherche de Goloupura », Befeo, 87, p. 79-107

Trouve G., 1933, Chronique Efeo 1933, Befeo, 33 (2), 1134 p.

\section{Le partenariat entre l'Inrap et l'Autorité pour la protection du Site et de l'Aménagement de la Région d'Angkor}

Ros Borath

Président de la commission nationale pour le Patrimoine mondial, directeur général adjoint de l'APSARA

U n partenariat entre l'Inrap et l'Apsara (Autorité pour la protection du site et l'aménagement de la région d'Angkor) a été initié en 2004 afin de permettre la première opération archéologique préventive de grande ampleur au Cambodge. Cette opération était financée par la Société concessionnaire de l'Aéroport de Siem Reap (filiale du groupe Vinci), aménageur du site de l'aéroport. De fait, le développement du tourisme entraîne des aménagements importants qu'il est nécessaire d'adapter au mieux sans atteindre à l'intégrité de ce patrimoine archéologique et architectural unique. À Angkor, le besoin d'étendre la capacité d'accueil de l'aéroport par la construction d'un nouveau terminal a été reconnu d'utilité publique. Cet important complexe aéroportuaire, existant avant l'inscription du site d'Angkor sur la liste du Patrimoine mondial de l'Unesco (décembre 1992), se retrouve aujourd'hui situé dans la zone $\mathrm{n}^{\circ} 1$, dite de sites monumentaux, méritant le plus haut degré de protection.
L'opération de diagnostic s'est déroulée de janvier à mai 2004 sur une superficie totale de 26 ha. Cette opération s'étant révélée très positive, l'Apsara a prescrit deux opérations de fouilles de sauvetage, de mai à juillet 2004, confiées à un agent de l'Inrap en collaboration avec des archéologues cambodgiens appartenant au département des Monuments et de l'Archéologie et des archéologues intégrés à l'unité d'Archéologie Préventive à la suite de cette expérience (cf. p. 86 article de Pierre Bâty). Ces fouilles ont concerné le temple de Trapeang Thlok, daté du XI ${ }^{\mathrm{e}}$ siècle et un habitat associé. Par ailleurs, l'importance des vestiges révélés par le diagnostic aux abords du temple de Prasat Trapeang Ropou a permis la prise de mesures conservatoires et le gel des terrains concernés, ainsi qu'une étude préliminaire pour la mise en valeur du site par l'unité de Gestion des abords. Les résultats de cette première fouille extensive et de ce premier diagnostic sont importants car ils permettent d'aborder un site dans toute sa complexité : un temple, un village, un espace foncier.

Outre la mise en ouvre et la réalisation de ces opérations, l'un des objectifs a été la formation des archéologues cambodgiens aux méthodes de l'archéologie préventive de grands travaux. La réussite de cette première opération a conduit nos deux établissements publics à se rapprocher par la signature d'une convention cadre de coopération internationale, d'une durée de trois ans, conformément à la politique de transfert de compétences soutenue par la France ainsi que par la Communauté internationale afin de conforter les efforts de l'Apsara.

\section{Le savoir-faire des topographes de l'Inrap dans les missions archéologiques françaises à l'étranger}

Patrick Deleuze

Inrap

$\mathrm{D}$ epuis la création de l'Afan, certains archéologues ou spécialistes associés, parmi lesquels des topographes, se sont engagés individuellement dans des missions à l'étranger, au gré des opportunités et de leurs travaux personnels. Étant moi-même topographe et ayant travaillé à l'Ifao, en Égypte, j'ai été chargé par le président et par le directeur général d'alors (Philippe Cuvillier et Alain Loiseau) d'officialiser et de cadrer une collaboration qui s'était installée de fait. À la création de l'Inrap, le président, Jean-Paul Demoule, et la directrice générale, Nicole Pot, ont intégré ces missions dans le cadre des projets d'actions scientifiques à l'étranger, sous la responsabilité de Nathan Schlanger.

À partir de 1997, sous la forme de prestations, plusieurs collègues de l'Afan ont participé à des activités de coopération culturelle qui s'inscrivaient dans l'esprit de partenariat nord-sud que la 